\title{
The potentially hazardous asteroid 2007CA19 as the parent of the $\eta$-Virginids meteoroid stream
}

\author{
P. B. Babadzhanov, G. I. Kokhirova, and Yu. V. Obrubov
}

\author{
Institute of Astrophysics, Academy of Sciences of the Republic of Tajikistan, 734042 Dushanbe, Tajikistan \\ e-mail: Kokhirova2004@mail.ru
}

Received 5 September 2014 / Accepted 13 April 2015

\begin{abstract}
The orbit of the potentially hazardous near-Earth asteroid 2007CA19 is classified as comet-like according to the Tisserand parameter with a value of $T_{j}=2.8$, therefore the object can be empirically considered as an extinct or dormant Jupiter-family comet. If 2007CA19 is really a former comet, it could have formed a meteoroid stream in the past in the period of its cometary activity. Investigation of the asteroid's orbital evolution shows that 2007CA19 is a quadruple-crosser of the Earth's orbit. Consequently, the meteoroid stream that is supposedly associated with the object can produce four meteor showers if, as expected, the perihelia arguments of the meteoroids are very distributed over the orbit. Theoretical radiants of the predicted showers were calculated using elements of the 2007CA19 osculating orbit that correspond to the positions of intersections with the Earth's orbit. A search for the predicted night-time showers has shown that the Northern and Southern $\eta$-Virginids can be associated to 2007CA19. Using the MOID IAU database, we identify two other daytime showers that can be associated with this asteroid. Thus, we confirm that $2007 \mathrm{CA} 19$ has an associated meteoroid stream that produces four active meteor showers in the Earth's atmosphere. This relationship supports the dynamical classification of the object and also points to the possibility of its cometary origin.
\end{abstract}

Key words. meteorites, meteors, meteoroids - minor planets, asteroids: general

\section{Introduction}

A population of small bodies in the solar system consists of meteoroids, comets, and asteroids. The meteoroid stream is a huge ensemble of meteoroids of a common origin, i.e. the meteoroids that were ejected from the same parent body. Owing to the relatively low velocities of ejection, the heliocentric orbits of stream's meteoroids are similar to the orbit of a parent. If the Earth passes through a meteoroid stream, a meteor shower can be observed in its atmosphere. These phenomena may occur in the night, as well as in the day time. To observe it, therefore, both the optical and radar techniques are used.

The parent bodies of meteoroids are comets and asteroids. Approaching the Sun, comet nuclei produce both a coma and the characteristic tails formed out of gases and dust. Asteroids, as a rule, do not show any such activity. These observed distinctions are conditioned by the difference between the physical nature of asteroids and comets. Comet nuclei are composed of frozen gases and dust particles (Whipple 1950), while asteroids are solid stony or iron-stony bodies.

Comets and asteroids may also be distinguished by their orbital features. For this purpose, several dynamical criteria are known (Kresak 1969). The Tisserand parameter, the values of which differ for the majority of comets and asteroids, is the one most frequently used. It is defined relative to Jupiter by the formulae (Kresak 1982; Kosai 1992):

$T_{j}=\frac{a_{j}}{a}+2\left[\frac{a}{a_{j}}\left(1-e^{2}\right)\right]^{0.5} \cos i$,

where $a, e$, and $i$ are the semimajor axis, eccentricity, and inclination of the object's orbit, respectively, and $a_{j}=5.2 \mathrm{AU}$ is the semimajor axis of the Jupiter orbit. According to Kresak (1982), the Tisserand parameter values range between 2.08 and 3.12 for comets and exceed the limit of 3.12 for asteroids. The value of $T_{j}$ at around 3 is still considered to be the boundary between asteroidal and cometary orbits (Jewitt 2012).

Currently, the powerful observing technique has led to a rapidly increasing number of small bodies being discovered. It becomes obvious that the distinctions between comets and asteroids cannot be clearly fixed. Today there are enough observational data confirming the existence of the sub-group of the small bodies that are moving on asteroid orbits, i.e. their Tisserand parameter $T_{j}>3$, but at the same time showing an activity that is like comets. These objects were called "main-belt comets" (Hsieh \& Jewitt 2006). Several mechanisms of mass loss were considered by Jewitt (2012) for 11 such bodies, and the term "active asteroids" was used for their designation, since some of the objects are not in the main belt. There are such known near-Earth objects as (3200) Phaethon, (2201) Oljato, and 107P/Wilson-Harrington in the set of active asteroids (Jewitt 2012).

Additionally, ambiguous difference between the physical properties of asteroids and comets is recognized at present. The hypothesis that there is a continuum between dark asteroids and comets in both the mineralogy and the chemical composition was suggested by Gounelle et al. (2008). The supposition was confirmed by the recent results obtained from the Stardust comet sample-return mission (Gounelle 2011).

The simulations of dynamical evolution of the carbonaceous microxenoliths, i.e. micrometeoroids with 100 and $200 \mu \mathrm{m}$ sizes, the parent bodies of which are Jupiter-family comets and carbonaceous asteroids, also strongly support this idea. Based on these results, Briani et al. (2011) conclude that the differences in structure and composition of carbonaceous asteroids and comets 
are less sharp than it was previously thought. Thus these results also point to the existence of an asteroids-comet continuum.

To date, more than 10000 asteroids have been discovered with perihelion distances below 1.3 AU. They were named as near-Earth asteroids (NEAs). Under the actions of planetary perturbations, orbits of the majority of NEAs can intersect the Earth's orbit.

The large portion of the NEAs population might be removed from the main asteroid belt that resides between Mars and Jupiter, owing to gravitational resonances with the major planets, mainly with Jupiter (Farinella et al. 1992). Such primordial asteroids are bodies of rocky or iron-stone composition. According to Opik (1963), another part of the NEAs are extinct or dormant comet nuclei; i.e., they represent the end state of usual comet nuclei after their multiple perihelion passages. They are composed of the conglomerates of frozen volatiles and solid particles covered by a thick dust mantle. This mantle prevents a sublimation of the volatiles under the solar heating; as a consequence, the out-gassing activity of these comets ceases and the development of a coma and tails stops. Jewitt (2012) considers dormant comets as inactive counterparts to the Jupiter-family comets, because their Tisserand parameter lies in the range $2 \leq T_{j}<3$.

While seen from a distance, dormant comets do not differ outwardly from primordial asteroids. But, owing to differences in the composition and structure, both groups represent different long-term dangers for the Earth as a consequence of possible impact. Since it is an ice-rich body, a dormant comet incoming into the Earth's atmosphere will produce the phenomena of superbolide and, probably, will explode in the atmosphere. Perharps, such scenario could apply in the case of the Tunguska event in Siberia in 1908. A strong rocky or metallic object like an asteroid penetrate the atmosphere deeper, and with low entry velocity, less than $25 \mathrm{~km} \mathrm{~s}^{-1}$, may reach the Earth's surface. The impact of an asteroid as large as $30 \mathrm{~m}$ in diameter, for example, occurs every few centuries, releasing energy of at least a few megatons of TNT. The most recent of these events was the entry into the Earth's atmosphere of the stone meteoroid with the initial mass of about ten kilotons and nearly $20 \mathrm{~m}$ in diameter on 15 February 2013 (Borovicka \& Spurny 2013; Brown 2013; Popova et al. 2014). As a result, the superfireball was produced that terminated by the meteorite fall in the Chelyabinsk area. Thus, the impacts of large asteroids, comets, and fireball-producing meteoroids with sizes of several tens of meters can have devastating consequences. Therefore, it is necessary to have a way to distinguish these groups, because the effects of a collision depend critically on the composition and structure of the objects.

Determining the geometric albedo of the NEAs is one of the ways to solve this problem. But a measurement of the albedo by the ground-based observations is a complicated task. Up to now albedo were determined only for a tiny number of the NEAs (Fernandez et al. 2001).

An alternative approach was elaborated with the assumption that those NEAs are dormant comets, which move on comet-like orbits and have the related meteoroid streams, formed at the period of activity of these comets (Babadzhanov \& Obrubov 1992). At the moment these meteoroid streams can produce the meteor showers observable on the Earth at the fixed periods. The existence of the meteor showers associated with some Earth-crossing asteroids is therefore the strongest evidence that these asteroids have a cometary origin, i.e. that they are dormant Jupiter-family comets at present.

The Taurid asteroid-meteoroid complex is a good example of the stream containing NEAs, which are really dormant comets. As a result of the Taurid complex investigations, more than 40 NEAs of cometary origin with sizes of few decameters were identified that are moving within the Taurid stream, alongside comet 2P/Encke (Asher et al. 1993; Babadzhanov 2001; Porubčan et al. 2004, 2006; Babadzhanov et al. 2008a; Rudawska et al. 2012a,b; Madiedo et al. 2013). The Quadrantids meteoroid stream is another such known set that is associated with comet 96P/Machholtz, a near-Earth object (NEO) 2003EH1, and possibly comet 1481 (Jenniskens 2004; Williams et al. 2004; Babadzhanov et al. 2008b; Neslusan et al. 2013). Using the criterion of dormant comet identification, new objects were revealed amongst the NEAs, which also are the constituent parts of the related meteoroid streams. For example, it turned out that the Piscids meteoroid stream has three associated NEOs 1997GL3, 2000PG3, and 2002JC9, which move within the stream and are dormant fragments of a larger comet-progenitor of this complex (Babadzhanov \& Williams 2007; Babadzhanov et al. 2008c). It was shown that the NEOs 2002JS2, 2002PD11, and 2003MT9 with very similar comet-like orbits are related to the $\iota$-Aquariids meteoroid stream, and this asteroid-meteoroid complex is the result of a cometary break-up (Babadzhanov et al. 2009). It was proved that the NEO 2004CK39 moving on a comet-like orbit is associated with the $v$-Virginids meteoroid stream and, very likely, is the dormant comet (Babadzhanov et al. 2012).

Scientific interest in dormant comets is caused by the need to identify them amongst the NEAs with the aim of applying data obtained for developing any mitigation strategy. Besides, the recognition of large bodies of cometary origin moving within meteoroid streams is very important for assessing the risk of an asteroid-comet impact.

After a brief description of the main concept of the formation and evolution of meteoroid streams, as well as the method and facilities, we present the result of finding the new potential dormant comet amongst the NEAs on the basis of its association with the meteoroid stream.

\section{Meteoroid streams and their meteor showers}

The formation of meteoroid streams as a result of the disintegration of cometary nuclei is now a fact. The relationship of comets and meteor showers has been studied well enough, and comets are considered to be the main source of meteoroids. This connection was confirmed by numerous observations, including the observed disintegration of comet 3D/Biela and the Andromedids meteor shower associated with it (Jenniskens 2004).

The problem of the interrelation of asteroids and meteoroid streams consists of the two important questions: 1) Do meteoroid streams exist that were formed by the destruction of real asteroids? 2) Do observable meteor showers exist that were produced by a meteoroid swarm with an asteroid origin?

The destruction of stony asteroids on a lot of small fragments can take place only once as the result of their mutual collisions. The tidal destruction under the attraction of major planets, rotational shedding, thermal fracture, or thermal stresses is much less probable. The collisions of the main belt asteroids occur continually. Craters on their surfaces and the existence of Hirayama families testify to this. However, it is doubtful that a lot of small fragments will be formed by the collisions. The ejection velocities even of the smallest fragments do not exceed $1 \mathrm{~km} \mathrm{~s}^{-1}$, so the orbits of fragments remain originally close to the orbit of a parent asteroid, and the existence of meteoroid streams with an asteroidal origin can be claimed.

The second question above needs for the mass and spatial density of fragments that were ejected due to the collision to 
be estimated. Because of very low probability of the collision, the multiple impacts can be excluded. Moreover, the spatial density will decrease rapidly under the planetary perturbations, so the mass and space density of meteoroid stream cannot be high and the lifetime of such a stream will be short. As a result, the hour number of any meteors from meteor showers of an asteroidal origin is low, and detecting such a meteoroid stream from observations becomes very complicated. Thus the probability of detecting the meteoroid stream of an asteroidal origin that produces the regular meteor showers is negligible. Though the possible existence of the meteoroid streams that are undoubtedly the result of asteroid fragmentation and are able to regularly deliver meteorites to the Earth was suggested by Halliday (1987), but this supposition still does not have any observational confirmation. As was established from observations, all known meteorites were only produced by the sporadic meteoroids with an asteroidal origin. The association of real asteroids and long-lived meteoroid streams remains questionable, but the reliable interrelation between comets and meteoroid streams may be applied without any doubt.

The following circumstances of the formation and evolution of cometary meteoroid streams would be taken into account while applying the method of determination of the dormant comets amongst the NEAs. Differences in the times, velocities, and points of the ejection of meteoroids from their parent comet, as well as the radiation pressure, determine an initial dispersion in the orbital elements of ejected meteoroids (Babadzhanov \& Obrubov 1992). The semimajor axes of orbits that define the meteoroids orbital periods get the largest variations. As a result, some of the ejected particles lag behind the parent comet, while others overtake it, thus producing a lengthened arc along the comet orbit. In a comparatively short time, the meteoroids spread along the entire parent's orbit and form a closed loop with non-uniform linear density for the particles (Hughes 1986; Williams 1995). The meteoroid stream is initially flat and thin, and is narrow at perihelion and is broadening at aphelion.

Owing to the initial dispersion of the semimajor axes $a$, the rates and cycles of variations in the inclinations $i$, eccentricities $e$, arguments of perihelia $\omega$, longitudes of the ascending nodes $\Omega$, and other orbital elements differ as well. With time, these differences cause an essential enhancement of the orbital dispersion within the meteoroid stream. The orbits of different meteoroids will be at different evolutionary stages that could be determined by their arguments of perihelia. Thus the stream's meteoroids occupy all evolutionary tracks of their parent body. This process considerably increases the size of the meteoroid stream.

From myriads meteoroids of the stream, only those can collide with the Earth that have orbits that intersect the Earth's orbit. It is only possible in the nodes of the meteoroid orbit, so the heliocentric distances of ascending $R_{\mathrm{a}}$ or descending $R_{\mathrm{d}}$ nodes of the orbit must be equal to the Earth's radius-vector, i.e. should be near $1 \mathrm{AU}$. This condition of selectivity may be written as

$R_{\mathrm{a}, \mathrm{d}}=\frac{a\left(1-e^{2}\right)}{1 \pm e \cos \omega} 1 \approx 1 \mathrm{AU}$

where the signs "+" and "-_" correspond to the ascending and descending nodes, respectively. The expression for calculating the $\omega$ values when the intersections of orbits occur and the eccentricity is known, may be obtained from Eq. (2):

$\cos \omega \approx \pm\left[\frac{a\left(1-e^{2}\right)-1}{e}\right]$
Equation (3) may have from one to eight roots. The most typical event is a case of quadruple crossings when the intersections occur at four different values of $\omega$. As a result, the meteoroid stream can produce a night-time shower with northern and southern branches at the pre-perihelion intersection with the Earth, and a twin daytime shower also with northern and southern branches at the post-perihelion intersection (Babadzhanov 2001).

\section{Research method}

We can conclude that some NEA probably has a cometary origin; that is to say, it is the extinct or dormant comet nucleus, if it moves on a comet-like orbit and has the associated meteoroid stream that produces the meteor showers observable on Earth.

The method of applying this criterion consists of several steps.

1. To determine the orbit type of the NEA under consideration, its Tisserand parameter $T_{j}$ (Eq. (1)) should be calculated. If $T_{j}<3.1$, then the orbit of given NEA can be classified as a comet-like.

2. The heliocentric distances of ascending $R_{\mathrm{a}}$ and descending $R_{\mathrm{d}}$ nodes of the present-day orbits of the majority of NEAs are not equal to $1 \mathrm{AU}$. Simple comparison of the NEAs orbits with the orbits of meteoroid streams is therefore not sufficient to establish their relationships. It is necessary to investigate the NEA orbital evolution and to find its osculating orbits at the moments of intersections with the Earth's orbit. To implement this task, the NEA's orbital evolution should be followed at least during one cycle of variation in the argument of perihelion.

3. Calculating the dynamical evolution yields the orbits of the NEA when it crosses the Earth's orbit. We intend that the meteoroid stream, possibly associated with this NEA, should produce meteor showers at these positions. Using the orbits found then, the theoretic geocentric radiants (right ascensions $\alpha_{\mathrm{g}}$ and inclinations $\left.\delta_{\mathrm{g}}\right)$ and velocities $V_{\mathrm{g}}\left(\mathrm{km} \mathrm{s}^{-1}\right)$ should be calculated, as well as the solar longitudes $L_{\odot}$ and corresponding dates of the meteor showers activity. Thus, we find the parameters of the theoretically predicted meteor showers that may be associated with this NEA. It should be noted that the theoretic night-time and daytime showers, as well as their northern and southern branches, are setting at this stage.

4. A search for the observed showers close to the predicted ones should be undertaken in the published catalogues of the meteor and fireball showers. The observations of individual meteors and fireballs, and the database of the IAU Meteor Orbit Data Center (MODC), should also be used.

5. If searching amongst the observations, all the theoretically predicted meteor showers interconnected with a NEA have been identified with active showers, then it can be concluded that this object very likely has a cometary origin and that it is now an inactive comet.

The similarity between the predicted and observed showers orbits may be evaluated by the $D_{S H}$ criterion of 
Table 1. Summary of the orbital and physical properties of the PHA 2007CA19.

\begin{tabular}{|c|c|c|c|c|c|c|c|c|c|c|c|c|c|}
\hline NEA & $\begin{array}{c}a \\
(\mathrm{AU})\end{array}$ & $e$ & $\begin{array}{c}q \\
(\mathrm{AU})\end{array}$ & $\begin{array}{c}Q \\
(\mathrm{AU})\end{array}$ & $\begin{array}{c}i \\
(\mathrm{deg}) \\
\end{array}$ & $\begin{array}{c}\Omega \\
(\mathrm{deg})\end{array}$ & $\begin{array}{c}\omega \\
(\mathrm{deg})\end{array}$ & $\begin{array}{c}\pi \\
(\mathrm{deg}) \\
\end{array}$ & $\begin{array}{c}R_{\mathrm{a}} \\
(\mathrm{AU})\end{array}$ & $\begin{array}{c}R_{\mathrm{d}} \\
(\mathrm{AU})\end{array}$ & $T_{j}$ & $H$ & $\begin{array}{c}d \\
(\mathrm{~km})\end{array}$ \\
\hline 2007CA19 & 2.787 & 0.827 & 0.483 & 5.11 & 9.62 & 174.8 & 97.6 & 272.4 & 0.96 & 0.75 & 2.8 & 17.6 & 0.90 \\
\hline
\end{tabular}

Southworth \& Hawkins (1963):

$$
\begin{aligned}
D_{S-H}^{2}= & \left(e_{2}-e_{1}\right)^{2}+\left(q_{2}-q_{1}\right)^{2}+\left(2 \sin \frac{i_{2}-i_{1}}{2}\right)^{2} \\
& +\sin i_{1} \sin i_{2}\left(2 \sin \frac{\Omega_{2}-\Omega_{1}}{2}\right)^{2} \\
& +\left[\left(\frac{e_{1}+e_{2}}{2}\right) 2 \sin \frac{\left(\Omega_{2}+\omega_{2}\right)-\left(\Omega_{1}+\omega_{1}\right)}{2}\right]^{2},
\end{aligned}
$$

where $q$ is the perihelion distance in AU, and subscripts 1 and 2 relate to the orbits that are being compared. The orbits are considered to be close if their value of $D_{S H} \leq 0.20$ (see e.g. Jenniskens 2006; Jopek \& Williams 2013).

Observed meteoroid orbits often do not show sufficient precision. Radar observations, providing the data on daytime showers, give especially low accuracy for these values. Along with the orbital elements, we have compared theoretical and observed geocentric radiants, velocities, and the dates of activity. We required that the difference in radiants not exceed \pm 10 deg in both right ascension and declination. The difference in geocentric velocities should be $\Delta V_{\mathrm{g}} \pm 5 \mathrm{~km} \mathrm{~s}^{-1}$, and the periods of activity may differ no more than \pm 15 days.

\section{The potentially hazardous asteroid 2007CA19}

The Apollo group Earth-crossing asteroid 2007CA19 was discovered on 10 February 2007 by the "Catalina Sky Survey" programme and was referred to Potentially Hazardous Asteroids (PHA) because of a close approach to the Earth at the distance 0.0002 AU (NEOP 2014). Its orbital and some of physical properties can be accessed at the "NEODyS" database (NEODyS 2013) and are summarized in Table 1. Here, the longitude of perihelion $\pi=\omega+\Omega$, the $T_{j}$ value, the absolute magnitude $H$, the equivalent diameter $d$ of the asteroid are given, along with the usual orbital elements. All orbital angular values are given in Equinox J2000.0.

As seen, the value of Tisserand parameter satisfies the condition $T_{j} \leq 3.1$, so the 2007CA19 orbit should be classified as comet-like, and the object may be considered as the candidate to an extinct or dormant Jupiter-family comet.

\subsection{Investigation of the orbital evolution of the PHA 2007CA19}

Secular variations in the orbit of 2007CA19 were calculated by the Halphen-Goryachev method (Goryachev 1937) taking perturbing actions of all major planets into account, including their mutual secular perturbations, during a time interval from 2000 $\mathrm{BC}$ to $2500 \mathrm{AD}$. The secular evolution of 2007CA19 is typical of the orbits of near-Earth quadruple-crosser objects. The time interval of 4000 years is slightly longer than one cycle of change of the argument of perihelion $\omega$. Variations in the argument and longitude of perihelion, as well as the inclination, eccentricity, and perihelion distance versus the time are given in Figs. 1-3.

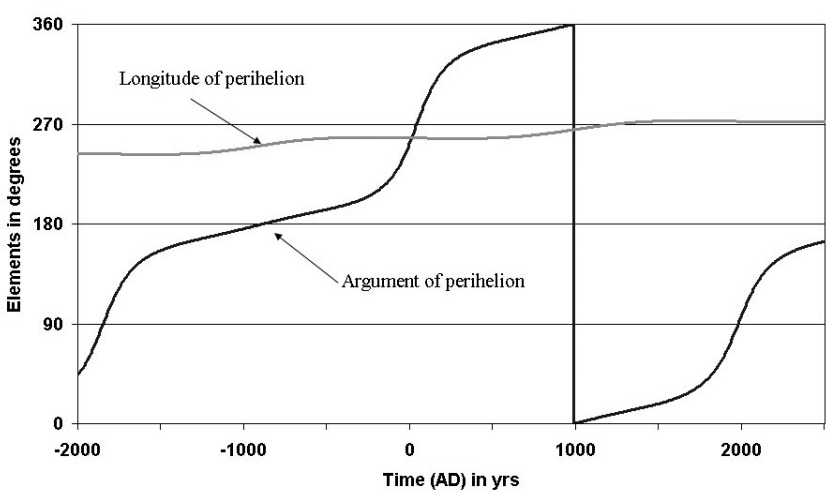

Fig. 1. Secular variations in the argument and longitude of perihelion of the PHA 2007CA19 orbit.

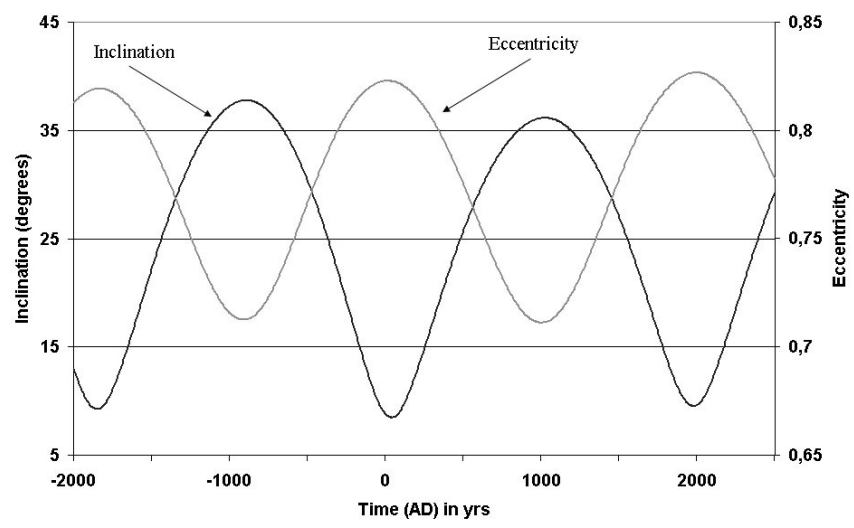

Fig. 2. Secular variations in the inclination and eccentricity of the PHA 2007CA19 orbit.

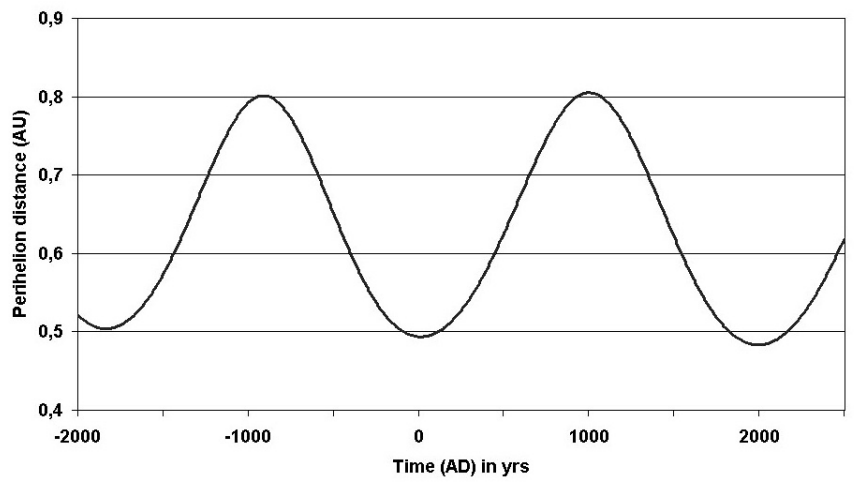

Fig. 3. Secular variations in the perihelion distance of the PHA 2007CA19 orbit.

The average rate of the argument of perihelion variation is equal to 9 deg per century, while for the longitude of perihelion, it is only 0.75 deg per century. Variations in the eccentricity and the inclination have a periodic character; moreover, 


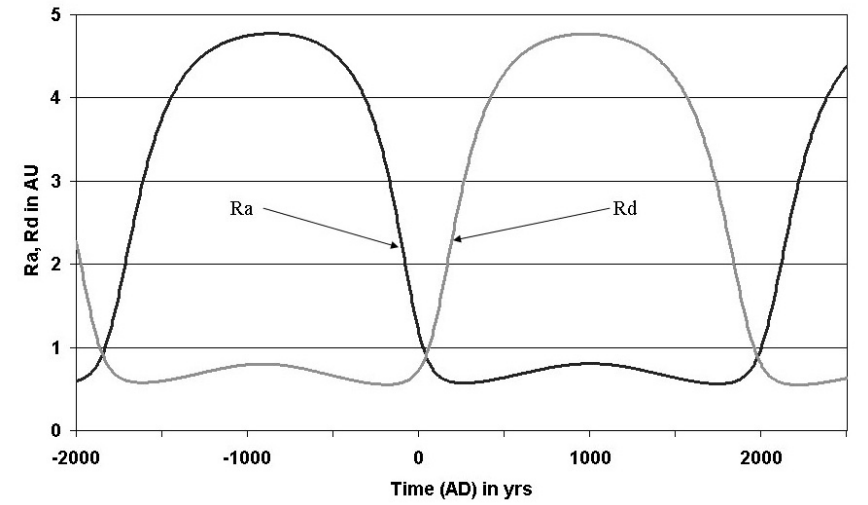

Fig. 4. Secular variations of the heliocentric distances of ascending $R_{\mathrm{a}}$ and descending $R_{\mathrm{d}}$ nodes versus the time of the PHA 2007CA19 orbit.

the period length is 4000 years. At this point, the eccentricity changes within $0.71-0.83$ and the inclination varies from 8.5 to $37.8 \mathrm{deg}$. The semimajor axis does not have secular perturbations and is assumed to be constant.

Secular variations in the argument of perihelion and the eccentricity cause secular variations in the ascending $R_{\mathrm{a}}$ and the descending $R_{\mathrm{d}}$ nodes of the orbit. These variations versus the time for the 2007CA19 orbit are presented in Fig. 4. As can be seen, the intersections with the Earth's orbit occur six times in the period under review and practically simultaneously at both the ascending and descending nodes. The difference between moments of crossings is not more than 40 years. After that, the potentially hazardous period is ending and the asteroid gets out from the area of possible impacts for about 2000 years.

The elements of the 2007CA19 orbit for the moments of intersections with the Earth's orbit are arranged in Table 2, where $T$ is the year of intersection of orbits.

Variations in the nodal heliocentric distances $R_{\mathrm{a}}$ and $R_{\mathrm{d}}$ lie in the range from 0.6 to $4.8 \mathrm{AU}$, and consequently, the orbit of 2007CA19 can cross not only the Earth's orbit, but the orbits of Venus and Mars as well. Moreover, this object is the quadruplecrosser for Mars and the octuple-crosser for Venus. Therefore, it may theoretically impact with these planets, as well as with the Moon. To provide the security of the civilization, possibly, considering the energy expenses, it will be more gainful to "organize" the impact of the object, for example, with Mars.

As noted above, the orbit of each stream's meteoroid corresponds to the different stages of dynamical evolution. The evolutionary stage can be characterized by the argument of perihelion. This element shows which part of the orbit is located over or under the ecliptic plane. This position exactly defines the rate and direction of variations in the inclination, eccentricity, and perihelion distance.

Variations in the heliocentric distances of ascending $R_{\mathrm{a}}$ and descending $R_{\mathrm{d}}$ nodes in dependence on the argument of perihelion $\omega$ of the 2007CA19 orbit are demonstrated by Fig. 5. As seen, 2007CA19 is a quadruple-crosser because its orbit intersects the Earth's orbit four times during one cycle of change of the argument of perihelion.

Thus, since a dispersion of the arguments of perihelions of meteoroids in the stream embraces whole range from 0 to $360 \mathrm{deg}$, then this stream will produce four meteor showers simultaneously. There are the northern and southern branches of the night-time shower, forming at the pre-perihelion intersection with the Earth, and the northern and southern branches of the daytime shower that are producing at the post-perihelion

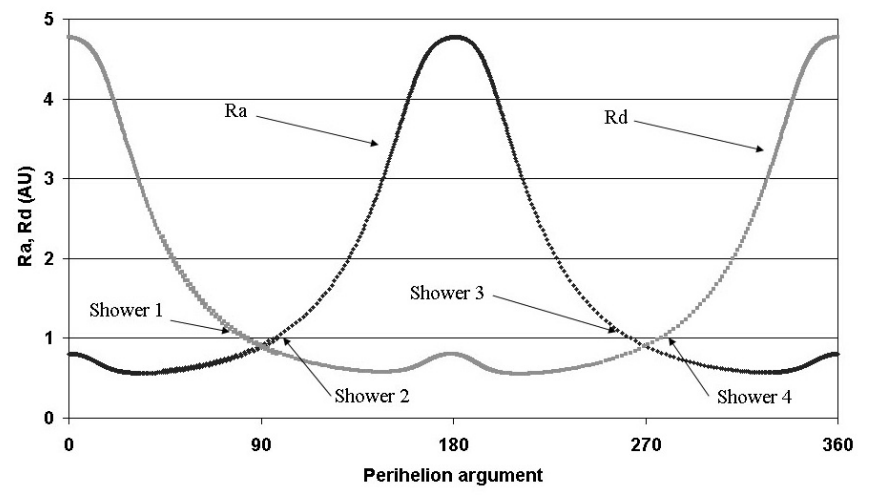

Fig. 5. Secular variations of the heliocentric distances of ascending $R_{\mathrm{a}}$ and descending $R_{\mathrm{d}}$ nodes versus the argument of perihelion $\omega$ of the PHA 2007CA19 orbit: shower 1 - northern branch of daytime shower (NDS), 2 - southern branch of night-time shower (SNS), 3 - southern branch of daytime shower (SDS), 4 - northern branch of night-time shower (NNS).

intersection (Fig. 5). Moreover, both branches of the same shower are active, approximately, at the same values of the solar longitude, while the night-time and daytime showers act at the different solar longitudes, which may theoretically differ until $180 \mathrm{deg}$.

\section{Meteor showers of the object 2007 CA19}

During 4500 years the argument of perihelion of 2007CA19 changed more than $360 \mathrm{deg}$, while the longitude of perihelion $\pi$ varied only on $30 \mathrm{deg}$ (Fig. 1). As far the rate of the longitude of perihelion variations is much less than the argument of perihelion variations rate (by the order), then a dispersion in the $\pi$ values of the meteoroids orbits increases slowly. To calculate theoretical radiants, the 2007CA19 orbits at the crossings' moments were used (Table 2), and the value of $\pi$ was assumed to be constant and equal to the observed longitude of perihelion: $\pi=$ $272.42 \mathrm{deg}$. The longitudes of ascending nodes $\Omega$ of the theoretic orbits were calculated by the expression $\Omega=272.42 \operatorname{deg}-\omega$.

The theoretic orbits and radiants of all four meteor showers associated with 2007 CA19 and denoted as NNS, SNS, NDS, and SDS, as well as the results of a computerized search for observed showers are given in Table 3. Four intersections just before 2000AD were used to calculate the features of the predicted showers are presented in Table 3: the elements of orbits $q, e, i, \Omega, \omega, \pi$; the solar longitudes $L_{\odot}$ at the maximum activity of the showers and their corresponding dates; the geocentric coordinates of radiants (right ascension $\alpha_{\mathrm{g}}$ and declination $\delta_{\mathrm{g}}$ ), the geocentric velocities $V_{\mathrm{g}}\left(\mathrm{km} \mathrm{s}^{-1}\right)$, the values of the $D_{S H}$ criterion. Finally, the same data for the observable showers and individual meteors or fireballs close to the predicted showers are arranged in Table 3, as are the catalogues, are indicated where they were found. The angular elements in Table 3 are given in Equinox J2000.0.

In Table 3 the catalogues used are denoted as S2 and S3 (Sekanina 1973, 1976), the results we used from the observations by the Prairie network are labelled as PN (McCrosky et al. 1978) and the database of the IAU Meteor Orbit Data Center as MODC. 
Table 2. Orbital elements of the PHA 2007CA19 at the moments of intersections with the Earth's orbit.

\begin{tabular}{rccccccccc}
\hline \hline$T$ & $\begin{array}{c}a \\
(\mathrm{AU})\end{array}$ & $e$ & $\begin{array}{c}q \\
(\mathrm{AU})\end{array}$ & $\begin{array}{c}i \\
(\mathrm{deg})\end{array}$ & $\begin{array}{c}\Omega \\
(\mathrm{deg})\end{array}$ & $\begin{array}{c}\omega \\
(\mathrm{deg})\end{array}$ & $\begin{array}{c}\pi \\
(\mathrm{deg})\end{array}$ & $\begin{array}{c}R_{\mathrm{a}} \\
(\mathrm{AU})\end{array}$ & $\begin{array}{c}R_{\mathrm{d}} \\
(\mathrm{AU})\end{array}$ \\
\hline 1865 BC & 2.787 & 0.819 & 0.504 & 9.28 & 159.35 & 83.98 & 243.33 & 0.845 & 1.003 \\
$1835 \mathrm{BC}$ & 2.787 & 0.819 & 0.504 & 9.29 & 147.85 & 95.39 & 243.24 & 0.993 & 0.851 \\
25 AD & 2.787 & 0.823 & 0.494 & 8.52 & 354.45 & 263.08 & 257.53 & 0.999 & 0.819 \\
60 AD & 2.787 & 0.822 & 0.495 & 8.54 & 340.03 & 277.40 & 257.43 & 0.816 & 1.009 \\
$1960 \mathrm{AD}$ & 2.787 & 0.826 & 0.484 & 9.61 & 191.69 & 81.80 & 272.56 & 0.791 & 1.002 \\
$2000 \mathrm{AD}$ & 2.787 & 0.827 & 0.483 & 9.62 & 174.86 & 97.56 & 272.42 & 0.991 & 0.796 \\
\hline
\end{tabular}

Table 3. Theoretic and observed orbital elements, geocentric radiants, and velocities of the meteor showers associated with the NEA $2007 \mathrm{CA} 19$ (J2000.0).

\begin{tabular}{|c|c|c|c|c|c|c|c|c|c|c|c|c|c|}
\hline $\begin{array}{l}\text { Showers, } \\
\text { fireballs }\end{array}$ & $\begin{array}{c}q \\
(\mathrm{AU})\end{array}$ & $e$ & $\begin{array}{c}i \\
\text { (deg) }\end{array}$ & $\begin{array}{c}\Omega \\
(\mathrm{deg})\end{array}$ & $\begin{array}{c}\omega \\
(\operatorname{deg})\end{array}$ & $\begin{array}{c}\pi \\
(\mathrm{deg})\end{array}$ & $\begin{array}{c}L_{\odot} \\
(\operatorname{deg})\end{array}$ & $\overline{\overline{\text { Date }}}$ & $\begin{array}{c}\alpha_{\mathrm{g}} \\
(\mathrm{deg})\end{array}$ & $\begin{array}{c}\delta_{\mathrm{g}} \\
(\mathrm{deg})\end{array}$ & $\begin{array}{c}V_{\mathrm{g}} \\
\left(\mathrm{km} \mathrm{s}^{-1}\right)\end{array}$ & $\overline{\overline{D_{S H}}}$ & $\begin{array}{l}\text { Cata- } \\
\text { logue }\end{array}$ \\
\hline NNS & 0.496 & 0.822 & 8.5 & 356.0 & 276.4 & 272.4 & 356.0 & Mar. 17 & 183.7 & 8.4 & 26.5 & & \\
\hline N.EVI & 0.495 & 0.707 & 3.7 & 358.0 & 282.4 & 280.4 & 358.0 & Mar. 19 & 185.8 & 2.8 & 23.0 & 0.18 & $\mathrm{~S} 2$ \\
\hline N.EVI & 0.502 & 0.703 & 11.3 & 350.0 & 281.8 & 271.8 & 350.0 & Mar. 11 & 183.0 & 13.8 & 23.5 & 0.13 & S3 \\
\hline 680306 & 0.389 & 0.860 & 3.7 & 345.7 & 287.4 & 273.1 & 345.7 & Mar. 6 & 176.7 & 5.5 & 29.3 & 0.14 & PN \\
\hline 710319 & 0.537 & 0.710 & 5.6 & 358.7 & 276.9 & 275.6 & 358.7 & Mar. 19 & 184.7 & 5.9 & 22.6 & 0.14 & PN \\
\hline SNS & 0.504 & 0.818 & 9.3 & 176.1 & 96.3 & 272.4 & 356.1 & Mar. 17 & 175.5 & -9.2 & 26.2 & & \\
\hline S.EVI & 0.499 & 0.706 & 2.6 & 171.1 & 101.8 & 272.9 & 351.1 & Mar. 12 & 176.1 & -1.2 & 22.9 & 0.13 & $\mathrm{~S} 3$ \\
\hline 640315 & 0.417 & 0.900 & 12.3 & 175.7 & 105.4 & 281.1 & 355.7 & Mar. 16 & 179.7 & -10.4 & 31.0 & 0.20 & PN \\
\hline 750228 & 0.415 & 0.830 & 0.4 & 159.7 & 107.1 & 266.8 & 339.7 & Feb. 29 & 169.2 & -4.8 & 28.0 & 0.13 & PN \\
\hline NDS & 0.485 & 0.826 & 9.6 & 190.6 & 81.8 & 272.4 & 190.6 & Oct. 4 & 189.4 & 6.8 & 26.9 & \multirow{3}{*}{0.05} & \multirow{3}{*}{ MODC } \\
\hline \multirow[t]{2}{*}{29 meteors } & 0.484 & 0.827 & 6.6 & 189.3 & 82.3 & 271.6 & 189.9 & Oct. 3 & 187.5 & 5.2 & 26.9 & & \\
\hline & \pm 0.020 & \pm 0.020 & \pm 0.8 & \pm 1.4 & \pm 3.1 & \pm 2.3 & & & \pm 1.1 & \pm 1.1 & \pm 0.4 & & \\
\hline SDS & 0.493 & 0.823 & 8.5 & 9.4 & 263.1 & 272.4 & 189.4 & Oct. 2 & 181.3 & -10.4 & 26.5 & \multirow{2}{*}{0.06} & \multirow{2}{*}{ MODC } \\
\hline 22 meteors & \pm 0.020 & \pm 0.010 & \pm 0.7 & \pm 1.4 & \pm 2.9 & \pm 2.6 & & & \pm 1.3 & \pm 1.1 & \pm 0.5 & & \\
\hline
\end{tabular}

\section{Discussion}

As seen from Table 3, the predicted night-time showers are identified well with the active northern and southern $\eta$-Virginids meteor showers denoted as N.EVI and S.EVI, respectively. Moreover, four fireballs detected by the Prairie network may also belong to these showers. The showers were established from the radar meteors observations by Sekanina (1973, 1976). Additionally, while analyzing the results of fireball networks observations, Terentjeva (1989) listed them as fireball showers. The $\eta$-Virginids shower was numbered as $00011 \mathrm{EVI}$ in the catalogue of Jenniskens (2006), as well as in the IAU Showers list.

For the predicted daytime showers, 29 meteors for the northern and 22 meteors for the southern branches were found among radar observations in the database of the IAU MODC. Their mean values with standard deviations are given in Table 3. According to the radiants positions and activity dates, these showers might be call the Daytime Northern and Southern October Virginids.

The values of $D_{S H}$ criterion given in Table 3 show a satisfactory coincidence of the orbital elements of the predicted and observed showers. A closeness of their radiant positions, velocities, and dates of activity also prove the association of the object 2007CA19 and the $\eta$-Virginids meteoroid stream.

The relation we found between 2007CA19 and four active and observed meteor showers, as well as for fireballs, confirms the dynamical classification of the object as an extinct comet. Moreover, the orbit of 2007CA19 presently corresponds to the night-time Southern $\eta$-Virginids meteor shower and, consequently, possible impact of 2007CA19 with the Earth will be characterized by the same parameters. The date of possible impact is 17 March, the geocentric velocity is $26.2 \mathrm{~km} \mathrm{~s}^{-1}$, and the equatorial coordinates of a point, from which it will be directed to the Earth, are $\alpha_{\mathrm{g}}=175.5 \mathrm{deg}$ (right ascension) and $\delta_{\mathrm{g}}=9.2 \mathrm{deg}$ (declination).

This evidence should not be considered as undoubted confirmation of any cometary nature of the object 2007CA19. On the basis of investigations of the dynamical evolution of NEAs, Fernandez et al. (2014) suggest that some of them, including 2007CA19, that move on stable orbits must be classified as primordial asteroids. Following their suggestion, comets Machholz, Encke, and many others are "real" asteroids according to their orbital evolution. These objects also have the orbits without the catastrophic changes, but their classification as comets is generally accepted. To approve certainly any supposition about the object's nature, it is necessary to examine it by the contact methods, for example by a lander, which allow investigating the composition and structure of the celestial body. As demonstrated by the ROSETTA mission, such a possibility exists and may be implemented.

If 2007CA19 is a real asteroid, i.e. a dense stony body, then it could be fragmented into a lot of debris under the tidal forces during the close encounters with major planets, by rotational shedding, thermal stresses, thermal fracture, or encounters with other asteroids and large meteoroids, etc. In any case, the meteoroid stream will be formed but, as was emphasized, the long stability of such stream is questionable.

\section{Conclusions}

Thus, the existence of the four active meteor showers produced by the $\eta$-Virginids meteoroid stream and the association of this stream with the NEA 2007 CA19 have been proved. 
This relation confirms a possible cometary origin for 2007CA19, which moves on a comet-like orbit. In this case, it can be concluded that either (i) the object 2007CA19 is a dormant parent Jupiter-family comet of the stream or (ii) this complex of nearEarth objects was formed as a result of the decay of a larger comet-progenitor and now consists of the $\eta$-Virginids meteoroid stream and the large extinct or dormant remnant of a parent. Furthermore, the possibly existing large fragments of the parent comet nucleus should be searched. That are also extinct at this stage, but may be found amongst the known NEAs and newly discovered ones.

Since the object 2007CA19 is the constituent part of the $\eta$-Virginids meteoroid stream, we concluded that the stream consists of both bodies with sizes from several tens of microns to one $\mathrm{km}$, as in the cases of the Taurids, Piscids, Scorpiids, etc. meteoroid streams.

This study allows it to conclude that the suggested method of identifying extinct comets amongst the NEAs is effective and yields the reliable results. However, to be more confident, it is necessary to intensify observations of the Virginids showers in order to obtain both more precise the dynamical features and the physical properties of meteoroids of the stream that is related to the very likely inactive comet 2007 CA 19 .

Acknowledgements. We would like to express our gratitude to the referee Dr. J.A. Fernandez for discussion and useful comments that improved the paper.

\section{References}

Asher, D. J., Clube, S. V. M., \& Steel, D. I. 1993, MNRAS, 264, 93

Babadzhanov, P. B. 2001, A\&A, 373, 329

Babadzhanov, P. B., \& Obrubov, Yu. V. 1992, Celest. Mech. Dyn. Astron., 54, 111

Babadzhanov, P. B., \& Williams, I. P. 2007, in Near-Earth objects our celestial neighbors: opportunity and risk, Proc. IAU Symp. 236, eds. A. Milani, G. B. Valsecchi, \& D. Vokrouhlicky (Cambridge University Press), 135

Babadzhanov, P. B., Williams, I. P., \& Kokhirova, G. I. 2008a, MNRAS, 386, 1436

Babadzhanov, P. B., Williams, I. P., \& Kokhirova, G. I. 2008b, A\&A, 479, 249

Babadzhanov, P. B., Williams, I. P., \& Kokhirova, G. I. 2008c, MNRAS, 386, 2271

Babadzhanov, P. B., Williams, I. P., \& Kokhirova, G. I. 2009, A\&A, 507, 1067

Babadzhanov, P. B., Williams, I. P., \& Kokhirova, G. I. 2012, MNRAS, 420, 2546

Borovicka, J., \& Spurny, P. 2013, in Meteoroids 2013 Abstracts (Poznan, Poland: A. M. University), 001

Briani, G., Morbidelli, A., Counelle, M., \& Nesvorny, D. 2011, Meteor. Planet. Sci., 46, 1863
Brown, P. 2013, in Meteoroids 2013 Abstracts (Poznan, Poland: A. M. University), 002

Farinella, P., Gonzi, R., Froeshle, Ch., \& Froeshle, Cl. 1992, in Asteroids, Comets, Meteors 1991 Conf., eds. A. Harris, \& E. Bowell, Lunar and Planetary Inst. (Houston: LPI), 167

Fernandez, Y. R., Jewitt, D., \& Shepard, S. S. 2001, ApJ., 553, L197

Fernandez, J. A., Sosa, A., Gallardo, T., \& Gutierrez, J. N. 2014, Icarus, 238, 1

Goryachev, N. N. 1937, Halphen's Method for Calculation of Planetary Secular Perturbations and its Application to Ceres, Krasnoe Znamya Tomsk

Gounelle, M. 2011, Elements, 7, 29

Gounelle, M., Morbidelli, A., Bland, P. A., et al. 2008, in The Solar System Beyond Neptune, eds. M. A. Barucci, H. Boehnhardt, D. P. Crukshank, \& A. Morbidelli (Tucson: Univ. Arizona Press), 525

Halliday, I. 1987, Icarus, 69, 550

Hsieh, H. H., \& Jewitt, D. 2006, Science, 312, 561

Hughes, D. W. 1986, in Asteroids, Comets, Meteors II, eds. C.-I. Lagerkvist, B. A. Lindblad, H. Lundstedt, \& H. Rickman (Uppsala Universitet Reprocentralen), 503

Jenniskens, P. 2004, AJ, 127, 3018

Jenniskens, P. 2006. Meteor Showers and Their Parent Comets (Cambridge, UK: Cambridge Univ. Press), 790

Jewitt, D. C. 2012, AJ, 143, 66

Jopek, T., \& Williams, I. P. 2013, MNRAS., 430, 2377

Kosai, H. 1992, Celest. Mech. Dyn. Astron., 54, 237

Kresak, L. 1969, Bull. Astr. Inst. Czechosl., 20, 177

Kresak, L. 1982, Bull. Astr. Inst. Czechosl., 33, 104

Madiedo, J. M., Trigo-Rodríguez, J. M., Williams, I. P., et al. 2013, MNRAS, 431, 2464

McCrosky, R. E. J., Shao, C. Y., \& Posen, A. 1978, Meteoritika, 37, 44

Neslusan, L., Kanuchova, Z., \& Tomko, D. 2013, A\&A, 551, A87

NEODyS 2013, http://newton.dm.unipi.it/neodys

NEOP 2014, http://neo.jpl.nasa.gov

Opik, E. 1963, Adv. Astron. Astrophys., 2, 219

Popova, O., Jenniskens, P., Shuvalov, V., et al. 2014, in Meteoroids 2013, Proc. Int. Astron. Conf., eds. T. J. Jopek, F. J. M. Rietmeijer, J. Watanabe, \& I. P. Williams (Poznan, Poland: A. M. University Press), 3

Porubčan, V., Kornos, L., \& Williams, I. P. 2004, Earth Moon Planets, 95, 697

Porubčan, V., Kornos, L., \& Williams, I. P. 2006, Contrib. Astron. Obs. Scalnate Pleso, 36, 103

Rudawska, R., Vaubaillon, J., \& Jenniskens, P. 2012a, EPSC2012, Madrid, Spain, 886

Rudawska, R., Vaubaillon, J., \& Jenniskens, P. 2012b, 2012LPICo 1667, 6222

Sekanina, Z. 1973, Icarus, 18, 253

Sekanina, Z. 1976, Icarus, 27, 265

Southworth, R. B., \& Hawkins, G. S. 1963, Smith. Contrib. Astrophys., 7, 261

Terentjeva, A. K. 1989, WGN, 17, 242

Whipple, F. L. 1950, ApJ, 113, 375

Williams, I. P. 1995, Earth Moon Planets, 68, 1

Williams, I. P., Ryabova, G. O., Baturin, A. P., \& Chernitsov, A. M. 2004 MNRAS, 355, 1171 